\title{
Frakturversorgung der Patella
}

Alexander Ellwein, Helmut Lill

\section{Einleitung}

Die Patella ist das größte menschliche Sesambein und in die Sehne des M. quadriceps eingelagert. Medial und lateral verlaufen aponeurotische Fasern, die als Retinacula den Reservestreckapparat bilden. Somit kann trotz Verletzung der Patella über den Reservestreckapparat eine Restfunktion im Kniegelenk erhalten bleiben [1]. Biomechanisch dient die Patella als Hypomochlion. Durch Verlagerung des Hebelarmes nach ventral wird die Muskelkraft des M. quadriceps um bis zu 30\% erhöht [2,3]. Beim Bergabgehen wirkt die patellofemorale Gelenkreaktion wie eine Bremsbacke, die Zug- in Kompressionskräfte umwandelt $[3,4]$.

Frakturen der Patella treten mit 1\% aller Frakturen selten auf. Insgesamt sind sie für etwa 3,5\% aller Frakturen der unteren Extremität verantwortlich [3,5]. Basierend auf schwedischen Registerdaten liegt ihre Inzidenz bei 1,26,1 pro 100000 Einwohner [6, 7]. Männer sind dabei etwa doppelt so häufig betroffen wie Frauen und der Altersgipfel liegt zwischen 20 und 50 Jahren. Während bei älteren Patienten häufiger Trümmerfrakturen auftreten, werden bei jüngeren Patienten vor allem Querfrakturen durch den Zug des M. quadriceps beobachtet. Als Hauptursache für Patellafrakturen gelten Verkehrsunfälle mit 78\%, gefolgt von Arbeitsunfällen (14\%) und häuslichen Unfällen (11\%). Aber auch iatrogene Ursachen wie Folge nach Knieprothesen Implantation (0,1-4,5\%) [8,9], Plastik des medialen patello-femoralen Ligamentes (MPFL) [10] oder Patellarsehnen-Transplantatentnahme mit Knochenblock $(0,2 \%)[11,12]$ sind beschrieben.

\section{Diagnostik}

Patienten, die sich mit einer Patellafraktur in der Notaufnahme vorstellen, präsentieren häufig eine ausgeprägte Kniegelenksschwellung mit Hämarthros. Es wird dabei versucht, das Knie in einer gestreckten Position ruhigzustellen. Bei erheblicher Frakturdislokation kann der Frakturspalt bereits palpabel oder eine Kranialisierung des proximalen Frakturfragmentes sichtbar sein. Im Rahmen der klinischen Untersuchung ist die Bewegungsprüfung des Kniegelenkes schmerzbedingt häufig nur eingeschränkt möglich. Dennoch gilt es, die aktive Streckfähigkeit des Kniegelenkes zu testen, da diese eine entscheidende Grundlage für die Indikation zur konservativen
Therapie darstellt. Obligat ist die klinische Überprüfung und Dokumentation des Gefäß-Nerven-Status.

Die radiologische Diagnostik beinhaltet zunächst ein Röntgen in 2 Ebenen (a.-p. und seitlich) sowie eine fakultative Patellatangentialaufnahme. Entsprechend den Leitlinien wird aktuell die routinemäßige Durchführung einer CT-Diagnostik prä- und postoperativ nicht empfohlen [13]. Anhand einer radiologischen Auswertung konnten Lazaro et al. jedoch zeigen, dass $88 \%$ der Patellafrakturen eine Beteiligung des distalen Pols aufweisen, die nur in $44 \%$ nativradiologisch zu erkennen ist [14]. Zudem hatte eine präoperative CT-Diagnostik in $49 \%$ Einfluss auf die Therapiestrategie. Postoperativ ist die sichere Beurteilung der Fixation aller Fragmente, der Implantatlage sowie einer möglichen verbliebenen Gelenkstufe durch ein alleiniges Nativröntgen ebenfalls eingeschränkt [15]. Aus diesem Grund empfehlen die Autoren insbesondere bei Mehrfragmentfrakturen die großzügige Indikation zur prä- und postoperativen CT-Diagnostik.

\section{Merke \\ Großzügige Indikation zur prä- und postoperativen CT-Diagnostik.}

\section{Konservative Therapie}

Einfache Frakturen mit Gelenkstufen $<2$ mm, erhaltener aktiver Streckfähigkeit des Kniegelenkes sowie stabiler Fraktursituation werden konservativ behandelt $[3,16]$. Im Rahmen der konservativen Therapie kann eine Punktion des Kniegelenkes zur Entlastung des Hämarthros sinnvoll sein. Die Mobilisation erfolgt unter schmerzadaptierter Vollbelastung in einer Bewegungsorthese mit Flexionslimitierung bis $30^{\circ}$ für die $1 .-2$. Woche, $60^{\circ}$ für die 3.-4. Woche und $90^{\circ}$ für die 5.-6. Woche. Bei fehlender Compliance kann die 6-wöchige Ruhigstellung in einer Streckorthese erforderlich sein, die jedoch schlechtere funktionelle Ergebnisse erwarten lässt. Radiologische Verlaufskontrollen werden im eigenen Vorgehen nach 1, 3 und 6 Wochen empfohlen. Sollte sich hier eine Frakturdislokation zeigen, besteht die Indikation zur operativen Versorgung.

\footnotetext{
Merke

Indikation zur konservativen Therapie bei einfachen Frakturen mit Gelenkstufen $<2$ mm, erhaltener Streckfähigkeit und stabiler Fraktursituation.
} 

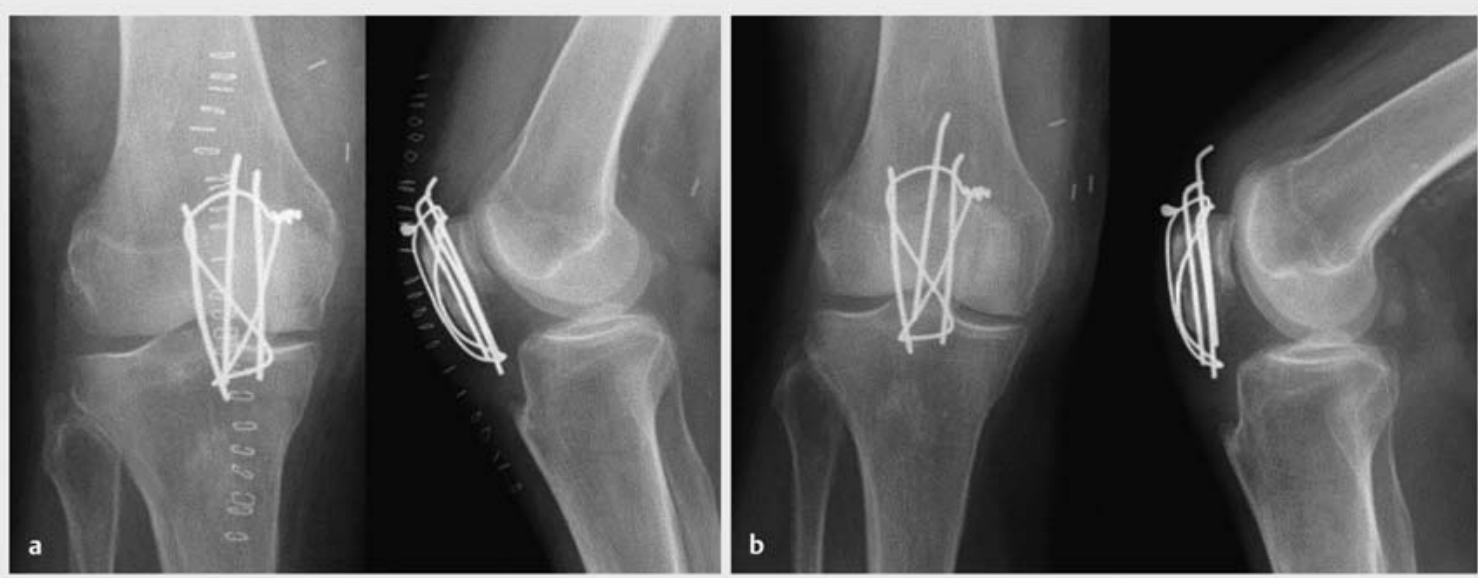

- Abb. 1 Patellafraktur rechts einer 75-jährigen Patientin. a Zuggurtungsosteosynthese. b K-Draht-Migration nach 6 Wochen.

\section{Operative Therapie}

Alle anderen Frakturen sind operativ zu versorgen. Im Rahmen der 8- bis 12-wöchigen Frakturheilung führt das Kniegelenk etwa 100000 Bewegungszyklen durch [3, 17]. Nachdem die Kraft auf das patellofemorale Gelenk bei $30^{\circ}$ Flexion dem Körpergewicht entspricht und bei $60^{\circ}$ Flexion auf das 4-Fache sowie bei $90^{\circ}$ Flexion auf das 8-Fache des Körpergewichtes ansteigt [18-20], wird in dieser Zeit eine Osteosynthese benötigt, die diese Kräfte ausreichend kompensiert.

Hinsichtlich des operativen Zugangsweges können sowohl ein zentraler oder parapatellarer Längsschnitt sowie eine Querinzision durchgeführt werden. Zur Entlastung des intraartikulären Hämatoms wird das Gelenk medial oder lateral mittels Arthrotomie eröffnet. Die patellare Blutversorgung erfolgt von inferomedial über einen extraossären Gefäßring, der von medial zur Patella zieht, sowie intraossär über Gefäße, die von inferior in den distalen Patellapol sowie in den ventralen Knochen einstrahlen [21-23]. Sofern die Frakturversorgung es zulässt, empfiehlt sich daher die laterale Arthrotomie zur Schonung der Gefäßversorgung mit Reduktion des Risikos postoperativer Nekrosen. Nach Reposition der Fraktur, die mit Kirschner-Drähten (K-Drähten) oder Repositionszange gehalten wird, kann diese digital über die Arthrotomie kontrolliert werden. Bei Trümmerfrakturen besteht die Möglichkeit der Erweiterung der Arthrotomie nach proximal und distal. So kann die stufenlose Reposition der Gelenkfläche unter Sicht kontrolliert werden. Im Falle eines distalen Polausrisses ist eine additive McLaughlinCerclage zu empfehlen, um entsprechende Zugkräfte zu kompensieren [24,25]. Aufgrund der obligaten Metallentfernung sowie der hohen Rate an Drahtbrüchen bei Draht-Cerclage werden heute trotz geringfügig erhöhter Fremdkörperreaktionen vermehrt Faden-Cerclagen (z. B. PDS-Kordel) verwendet [26].

\section{Merke}

Die laterale Arthrotomie ist aufgrund des Erhalts der Blutversorgung zu bevorzugen.

\section{Zuggurtungsosteosynthese}

Die Zuggurtungsosteosynthese stellt für viele weiterhin die Standardversorgung dar, vor allem in der operativen Versorgung von Patellaquerfrakturen. Hierbei werden 2 parallele K-Drähte senkrecht zur Fraktur eingebracht. Um die K-Draht-Enden wird eine 8-förmige Cerclage angelegt und die K-Draht-Enden über der Cerclage im Knochen versenkt. Die höchste Stabilität bietet diese Versorgung unter Verwendung zweier gegenüberliegender Drahtzwirbel [4]. Zur weiteren Verbesserung der Stabilität kann die 8-förmige Cerclage mit einer zirkulären Cerclage kombiniert werden. Die alleinige zirkuläre Cerclage kann die Distraktionskräfte nicht ausreichend neutralisieren [3].

Prinzip der Zuggurtungsosteosynthese ist die Umwandlung von Zug- in Kompressionskräfte [27]. Labitzke konnte jedoch in einer Vektoranalyse zeigen, dass es bei aktiver Streckung zwar an der Vorderseite zur Kompression, artikularseitig jedoch zu einem Öffnen des Frakturspaltes kommt [28]. Aktuellere biomechanische Untersuchungen stützen diese Aussage und bezeichnen die Zuggurtungsosteosynthese weniger als dynamische, sondern als statische Fixation [29].

Weitere Limitationen der Zuggurtungsosteosynthese sind sowohl in der unzureichenden Stabilisierung von Mehrfragmentfrakturen als auch in der hohen Komplikationsrate (22-53\%) zu sehen [30,31]. Implantatbedingte Komplikationen wie Cerclagen-/K-Draht-Migrationen (6$20 \%$ ) [32] ( A Abb. 1), Implantatbrüche (37\%) und -lockerungen $(36 \%)[33,34]$ stehen hierbei im Vordergrund. Aufgrund der instabilen Osteosynthese bei Implantatver- 

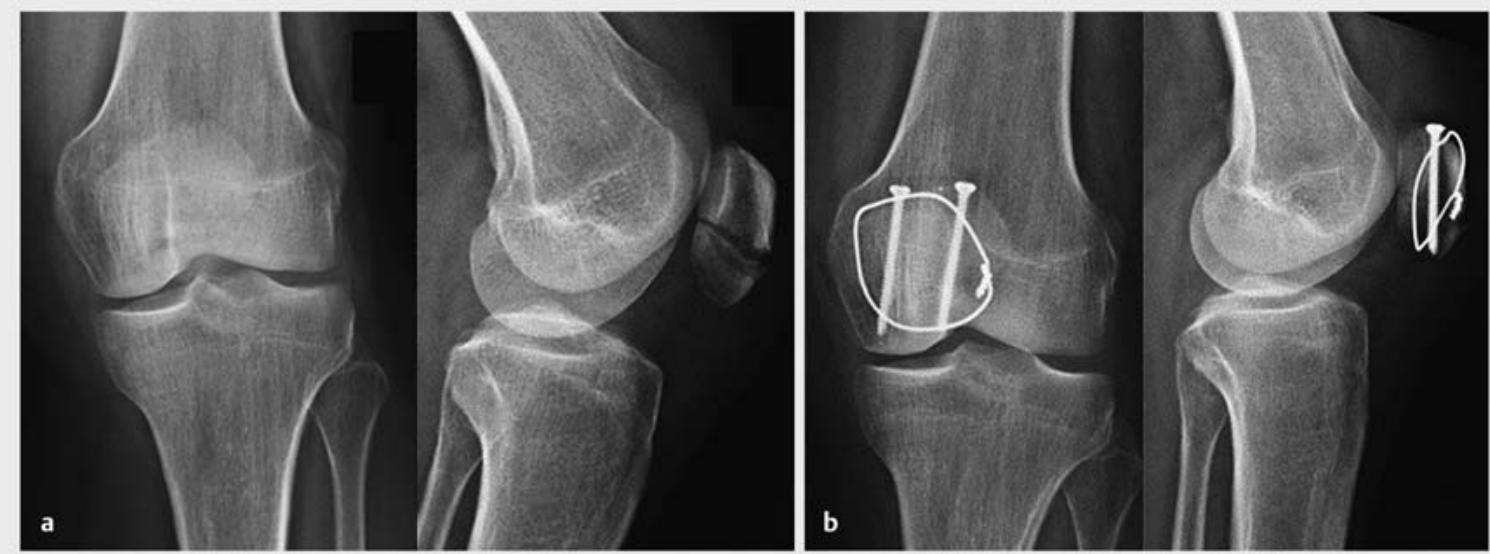

Abb. 2 Patellaquerfraktur einer 63-jährigen Patientin. a Traumabilder. b Schraubenosteosynthese mit additiver Äquatorial-Cerclage.

sagen werden sekundäre Frakturdislokationen in 22-30\% der Fälle beobachtet [31,34]. Die Lage der K-Drähte sowie der Cerclage habe dabei einen relevanten Einfluss. Eine zentrale Lage der K-Drähte im Knochen im Vergleich zu einer oberflächlichen Lage ist mit einer höheren Stabilität bei weniger Repositionsverlust vergesellschaftet [35]. Zudem treten Repositionsverluste und Implantatbrüche seltener auf, wenn die Zuggurtung dem Knochen eng anliegt [35]. Die häufigsten Komplikationen sind jedoch durch das Implantat bedingte Weichteilirritationen [31, 35-37], die in etwa der Hälfte der Fälle eine Materialentfernung erfordern [30,31, 35, 36, 38].

\section{Merke}

Implantatbedingte Weichteilirritationen machen in etwa der Hälfte der Fälle eine Metallentfernung nach Zuggurtungsosteosynthese erforderlich.

Hinsichtlich des klinischen Ergebnisses klagen 30-70\% der Patienten über persistierende Beschwerden [38], 5$37 \%$ weisen zudem funktionelle Einschränkungen auf [38]. Dies führt in bis zur Hälfte der Fälle zu einem allenfalls mäßigen bis schlechten Ergebnis [32, 33, 38, 39].

\section{Schraubenosteosynthese}

Durch eine verbesserte Stabilität kann durch die Schraubenosteosynthese das Risiko sekundärer Dislokationen reduziert werden [40]. Mindestens zwei Schrauben werden senkrecht zur Frakturebene eingebracht. Insbesondere einfache Querfrakturen eignen sich für diese Versorgung. Weitere kleinere Fragmente können ggf. über zusätzliche freie Schrauben fixiert werden. Zur weiteren Stabilisierung kann eine Äquatorial-Cerclage sinnvoll sein ( $\triangleright$ Abb. 2).

\section{Kanülierte Schraubenosteosynthese mit Zuggurtungsosteosynthese}

Die Schraubenosteosynthese kombiniert mit einer Zuggurtungsosteosynthese vereint die Vorteile beider Verfahren. Kanülierte Schrauben werden senkrecht zur Fraktur eingebracht. Durch die Schrauben wird der Draht für die Zuggurtung geführt, der vor der Patella in der klassischen 8er-Tour fixiert wird. Durch die Kombination beider Verfahren erhöhte sich die biomechanische Stabilität im Vergleich zu der jeweils alleinigen Versorgung [40]. Trotz der verbesserten Stabilität ist die adäquate Fixation von Mehrfragmentfrakturen weiterhin eingeschränkt. Zudem bleibt das Problem der Weichteilirritationen durch die Zuggurtung mit entsprechenden Revisionsraten ungelöst. Der Einsatz eines Faden-Tapes anstatt der Draht-Cerclage kann hier eine mögliche Lösung sein. Klinische und biomechanische Studien liegen hierzu jedoch noch nicht vor.

\section{Marknagelosteosynthese}

Unter dem Wissen der hohen Rate an Metallentfernungen nach Zuggurtungsosteosynthese sowie der unzureichenden Versorgung von Mehrfragmentfrakturen durch Zuggurtung und Schraubenosteosynthese haben Gehr und Friedl eine Marknagelosteosynthese entwickelt. Der XS-Nagel ${ }^{\circledR}$ (Fa. Intercus, Aarau, Schweiz) wird intramedullär und senkrecht zur Hauptfrakturzone eingebracht [39]. Der zentrische Kraftträger ermöglicht eine gleichmäßige Kompression über die gesamte Frakturzone. Die Fixation der einzelnen Frakturfragmente erfolgt über 4-11 KDrähte, die im $90^{\circ}$-Winkel zum Nagel eingebracht werden. Biomechanisch ist der XS-Nagel der Zuggurtungsosteosynthese überlegen, sodass aufgrund der verbesserten Stabilität die Autoren eine sofortige postoperative Vollbelastung erlauben. Im Kurzzeit-Follow-up nach 

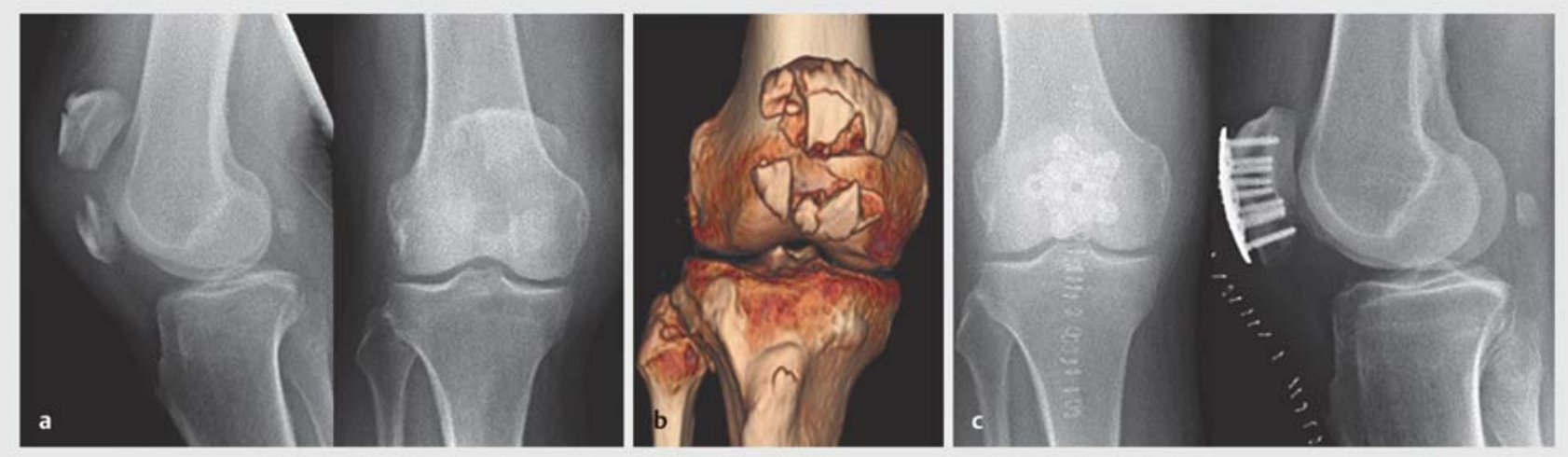

- Abb. 3 Patellamehrfragmentfraktur (AO C3) eines 73-jährigen Patienten. a Traumabilder. b CT mit 3-D-Rekonstruktion. c Winkelstabile Plattenosteosynthese (Star Plate).

durchschnittlich 4 Monaten zeigten 13 von 15 Patienten gute bis sehr gute Ergebnisse. Lediglich 2 Patienten wiesen ein Beugedefizit von mehr als $30^{\circ}$ im Vergleich zur Gegenseite auf, wobei beides Revisionseingriffe nach Versagen einer Zuggurtungsosteosynthese waren. Im klinischen Alltag hat sich diese Versorgung jedoch bisher nicht durchgesetzt.

\section{Winkelstabile Plattenosteosynthese}

Wild et al. haben als Erste den Einsatz einer winkelstabilen Plattenosteosynthese an der Patella beschrieben [41-45]. Diese polyaxiale Platte wird bilateral an der medialen und lateralen Patellakante angelegt. Biomechanisch ist diese Plattenosteosynthese (2396 N) sowohl der Zuggurtungsosteosynthese $(625 \mathrm{~N})$ als auch der Kombinationsosteosynthese aus kanülierten Schrauben und Zuggurtung (1015 N) überlegen. Klinisch zeigt die Platte nach einem Jahr durchweg gute bis sehr gute Ergebnisse [46]. Zudem liegt die Komplikationsrate lediglich bei $10 \%$ und Metallentfernungen sind nur noch bei $25 \%$ der Patienten erforderlich. Jedoch sehen die Autoren Einschränkungen in der Versorgung von Mehrfragmentfrakturen, da nicht alle Fragmente sicher gefasst werden können.

\section{Merke}

Winkelstabile Implantate weisen eine höhere Stabilität auf, die eine sofortige Vollbelastung erlaubt.

Die SuturePlate ${ }^{\mathrm{TM}}$ (Fa. Arthrex, Naples, Florida, USA) ist eine Alternative, die zusätzlich zur Versorgung von Mehrfragmentfrakturen geeignet ist. Die Platte wird ventral auf die Patella aufgelegt und mit monokortikalen Schrauben winkelstabil fixiert. Einfache Querfrakturen oder Polfrakturen werden mit einer pfeilförmigen Platte (Arrow Plate) versorgt. Aufgrund multipler Schraubenlöcher wird für Mehrfragmentfrakturen eine sternförmige Platte (Star Plate) empfohlen ( $\bullet$ Abb.3). Biomechanisch zeigt die
SuturePlate signifikante Vorteile gegenüber der Zuggurtungsosteosynthese [47]. Die verbesserte Primärstabilität ermöglicht eine sofortige Vollbelastung, welche die Rehabilitation und Rückkehr in den Alltag beschleunigt [15]. Sechs Monate postoperativ sind durchweg gute bis sehr gute klinische Ergebnisse beschrieben, bei $97 \%$ des gesunden Bewegungsumfanges [15]. Zudem zeigt sich mit $12 \%$ eine ebenfalls geringe Komplikationsrate.

\section{Merke}

Die Star Plate ermöglicht eine stabile Fixation von Mehrfragmentfrakturen durch multiple Schraubenlöcher.

\section{Nachbehandlung}

Klassischerweise werden Patellafrakturen unter Teilbelastung sowie Flexionslimitierung über 6 Wochen nachbehandelt. Im eigenen Vorgehen wird die Flexion für 2 Wochen auf $30^{\circ}$ limitiert, für weitere 2 Wochen auf $60^{\circ}$ und weitere 2 Wochen auf $90^{\circ}$. Moderne winkelstabile Implantate zeigen eine deutlich höhere Primärstabilität, sodass hier eine direkte Vollbelastung erlaubt ist. Zudem scheint man auf eine Flexionslimitierung möglicherweise verzichten zu können. Radiologische Verlaufskontrollen sind direkt postoperativ und nach 6 Wochen durchzuführen. Eine Implantatentfernung ist nicht obligat und muss individuell entschieden werden. Die Autoren empfehlen, das Implantat für mindestens 6 Monate in situ zu belassen. Bei Unklarheit hinsichtlich der knöchernen Durchbauung sollte eine CT angestrebt werden.

\section{Fazit für die Praxis}

- Einfache, undislozierte Frakturen mit erhaltener Streckfähigkeit im Kniegelenk, ohne wesentliche Frakturdislokation $(<2 \mathrm{~mm}$ ) und bei stabiler Fraktursituation sind konservativ zu behandeln. 
- Die Komplikationsrate und Revisionseingriffe nach Zuggurtungsosteosynthese sind hoch, bei nicht zufriedenstellenden klinischen Ergebnissen.

- Einfache Querfrakturen können stabil mittels Schraubenosteosynthese versorgt werden.

- Die Marknagelosteosynthese ist ein vielversprechendes Konzept, hat sich jedoch im klinischen Alltag nicht durchgesetzt.

- Winkelstabile Plattenosteosynthesen zeigen biomechanisch eine hohe Stabilität, sodass eine sofortige Vollbelastung möglich ist. Die klinischen Ergebnisse sind zudem vielversprechend.

- Die Star Plate bietet eine gute Möglichkeit der Versorgung von Mehrfragmentfrakturen aufgrund ihrer multiplen Schraubenlöcher.

\section{Interessenkonflikt}

Helmut Lill ist Consultant für Arthrex GmbH. Alexander Ellwein hat Referentenhonorare von Arthrex GmbH erhalten.

\section{Autorinnen/Autoren}

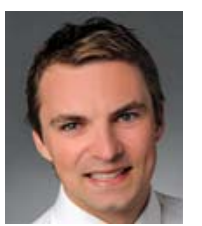

\section{Alexander Ellwein}

Dr. med., Facharzt, Department Schulter-, Knie- und Sportorthopädie, DIAKOVERE Annastift, Orthopädische Klinik der Medizinischen Hochschule Hannover

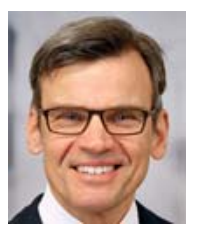

\section{Helmut Lill}

Prof. Dr. med., Chefarzt, Klinik für Orthopädie und Unfallchirurgie, DIAKOVERE Friederikenstift Hannover

Korrespondenzadresse

\section{Dr. Alexander Ellwein}

Department Schulter-, Knie- und Sportorthopädie DIAKOVERE Annastift, Orthopädische Klinik der

Medizinischen Hochschule

Anna-von-Borries-Straße 1-7

30625 Hannover

Tel.: $0511 / 5354-0$

Fax: 0511/5354-688

alexander.ellwein@diakovere.de

\section{Literatur}

[1] Galla M, Lobenhoffer P. Patella fractures. Chirurg 2005; 76: 987-997. doi:10.1007/s00104-005-1081-3

[2] Kaufer H. Mechanical function of the patella. J Bone Joint Surg Am 1971; 53: 1551-1560

[3] Wild M, Windolf], Flohé S. [Fractures of the patella]. Unfallchirurg 2010; 113: 401-411. doi:10.1007/s00113-010-1768-x

[4] Koval KJ, Kim YH. Patella fractures. Evaluation and treatment. Am J Knee Surg 1997; 10: 101-108
[5] Dietz SO, Hessmann MH, Gercek E et al. Patella fracture. Oper Orthop Traumatol 2009; 21: 206-220. doi:10.1007/s00064009-1708-5

[6] van Staa TP, Dennison EM, Leufkens HG et al. Epidemiology of fractures in England and Wales. Bone 2001; 29: 517-522

[7] Yang NP, Chan CL, Yu IL et al. Estimated prevalence of orthopaedic fractures in Taiwan - A cross-sectional study based on nationwide insurance data. Injury 2010; 41: 1266-1272. doi:10.1016/j.injury.2010.05.025

[8] Sheth NP, Pedowitz DI, Lonner JH. Periprosthetic patellar fractures. J Bone Joint Surg Am 2007; 89: 2285-2296. doi:10.2106/JBJS.G.00132

[9] Meding JB, Fish MD, Berend ME et al. Predicting patellar failure after total knee arthroplasty. Clin Orthop Relat Res 2008; 466: 2769-2774. doi:10.1007/s11999-008-0417-y

[10] Lippacher S, Reichel H, Nelitz M. [Patella fracture after patellar stabilization]. Orthopade 2010; 39: 516-518. doi:10.1007| s00132-010-1609-1

[11] Lee GH, McCulloch P, Cole B] et al. The incidence of acute patellar tendon harvest complications for anterior cruciate ligament reconstruction. Arthroscopy 2008; 24: 162-166. doi:10.1016/j.arthro.2007.08.020

[12] Benner RW, Shelbourne KD, Freeman $\mathrm{H}$. Infections and patellar tendon ruptures after anterior cruciate ligament reconstruction: a comparison of ipsilateral and contralateral patellar tendon autografts. Am J Sport Med 2011; 39: 519-525. doi: $10.1177 / 0363546510388163$

[13] Arbeitsgemeinschaft der Wissenschaftlichen Medizinischen Fachgesellschaften. Leitlinie der Deutschen Gesellschaft für Unfallchirurgie - Patellafraktur (2008). AWMF online. Im Internet: https://www.awmf.org/uploads/tx_szleitlinien/012-017I_ Patellafraktur_2014-06.pdf; Stand: 11.01.2019

[14] Lazaro LE, Wellman DS, Pardee NC et al. Effect of computerized tomography on classification and treatment plan for patellar fractures. J Orthop Trauma 2013; 27: 336-344. doi:10.1097/BOT.0b013e318270dfe7

[15] Ellwein A, Lill H, Jensen G et al. Die Plattenosteosynthese der Patellafraktur - Technik und erste Ergebnisse einer prospektiven Studie. Unfallchirurg 2017; 120: 753-760. doi:10.1007/ s00113-016-0213-1

[16] Wurm S, Augat P, Bühren V. Winkelstabile Plattenosteosynthese der Patella. Trauma Berufskrankh 2012; 14: 147-151. doi:10.1007/s10039-011-1815-z

[17] Bostrom MP, Asnis SE, Ernberg JJ et al. Fatigue testing of cerclage stainless steel wire fixation. J Orthop Trauma 1994; 8: 422-428

[18] Kettelkamp D. Clinical implications of knee biomechanics. Arch Surg 1973; 107: 406-410

[19] Oestern S, Varoga D, Lippross S et al. [Patella dislocation]. Unfallchirurg 2011; 114: 345-358. doi:10.1007/s00113-0112012-z

[20] Outerbridge R, Dunlop J. The problem of chondromalacia patellae. Clin Orthop Relat Res 1975; (110): 177-196

[21] Scapinelli R. Blood supply of the human patella. Its relation to ischaemic necrosis after fracture. J Bone Joint Surg Br 1967; 49: 563-570

[22] Schüttrumpf JP, Behzadi C, Balcarek $\mathrm{P}$ et al. Radiologically hyperdense zones of the patella seem to be partial osteonecroses subsequent to fracture treatment. \ Knee Surg 2013; 26: 319-326. doi:10.1055/s-0033-1336595

[23] Lazaro LE, Wellman DS, Klinger CE et al. Quantitative and qualitative assessment of bone perfusion and arterial contributions in a patellar fracture model using gadolinium-enhanced mag- 
netic resonance imaging: a cadaveric study. J Bone Joint Surg Am 2013; 95: e1401-e1407. doi:10.2106/JBJS.L.00401

[24] Ahrberg A, Josten C. [Augmentation of patella fractures and patella tendon ruptures with the McLaughlin-Cerclage]. Unfallchirurg 2007; 110: 685-690. doi:10.1007/s00113-007$1269-8$

[25] McLaughlin H. Repair of major tendon ruptures by buried removable suture. Am J Surg 1947; 74: 758-764

[26] Kasten P, Schewe B, Maurer F et al. Rupture of the patellar tendon: a review of 68 cases and a retrospective study of 29 ruptures comparing two methods of augmentation. Arch Orthop Trauma Surg 2001; 121: 578-582

[27] Müller M, Allgöwer M, Schneider R, Willenegger $H$. Manual of internal Fixation: Techniques recommended by the AO-ASIF. Heidelberg: Springer; 1991

[28] Labitzke R. Proper and improper tension band fixation exemplified by patellar fracture. Chirurg 1997; 68: 638-642. doi: $10.1007 / \mathrm{s} 001040050245$

[29] Zderic I, Stoffel K, Sommer $C$ et al. Biomechanical evaluation of the tension band wiring principle. A comparison between two different techniques for transverse patella fracture fixation. Injury 2017; 48: 1749-1757. doi:10.1016/j.injury.2017. 05.037

[30] Smith ST, Cramer KE, Karges DE et al. Early complications in the operative treatment of patella fractures. J Orthop Trauma 1997; 11: 183-187

[31] Chiang CC, Chen WM, Jeff Lin CF et al. Comparison of a minimally invasive technique with open tension band wiring for displaced transverse patellar fractures. J Chin Med Assoc 2011; 74: 316-321. doi:10.1016/j.jcma.2011.05.008

[32] Mehdi M, Husson JL, Polard JL et al. Treatment results of fractures of the patella using pre-patellar tension wiring. Analysis of a series of 203 cases. Acta Orthop Belg 1999; 65: 188-196

[33] Hung LK, Chan KM, Chow YN et al. Fractured patella: operative treatment using the tension band principle. Injury 1985; 16 : 343-347

[34] Wild M, Khayal T, Miersch D et al. [Dynamic cerclage wiring of patellar fractures. Complications and midterm functional results]. Unfallchirurg 2008; 111: 892-897. doi:10.1007/ s00113-008-1505-x

[35] Hsu KL, Chang WL, Yang CY et al. Factors affecting the outcomes of modified tension band wiring techniques in transverse patellar fractures. Injury 2017; 48: 2800-2806. doi:10.1016/j.injury.2017.10.016

[36] Mao N, Liu D, Ni H et al. Comparison of the cable pin system with conventional open surgery for transverse patella fractures. Clin Orthop Relat Res 2013; 471: 2361-2366. doi:10.1007/s11999-013-2932-8
[37] Luna-Pizarro D, Amato D, Arellano F et al. Comparison of a technique using a new percutaneous osteosynthesis device with conventional open surgery for displaced patella fractures in a randomized controlled trial. J Orthop Trauma 2006; 20 : 529-535. doi:10.1097/01.bot.0000244994.67998.1b

[38] Levack B, Flannagan JP, Hobbs S. Results of surgical treatment of patellar fractures. J Bone Joint Surg Br 1985; 67: 416-419

[39] Gehr J, Friedl W. Problems in osteosynthesis of patella fractures with the $\mathrm{AO}$ tension belt and consequences for new implants. The XS nail. Chirurg 2001; 72: 1309-1317

[40] Carpenter JE, Kasman RA, Patel $\mathrm{N}$ et al. Biomechanical evaluation of current patella fracture fixation techniques. J Orthop Trauma 1997; 11: 351-356

[41] Thelen S, Betsch M, Schneppendahl J et al. Fixation of multifragmentary patella fractures using a bilateral fixed-angle plate. Orthopedics 2013; 36: e1437-e1443. doi:10.3928/ 01477447-20131021-29

[42] Thelen S, Schneppendahl J, Baumgärtner R et al. Cyclic longterm loading of a bilateral fixed-angle plate in comparison with tension band wiring with K-wires or cannulated screws in transverse patella fractures. Knee Surg Sports Traumatol Arthrosc 2013; 21: 311-317. doi:10.1007/s00167-012-1999-1

[43] Thelen S, Schneppendahl J, Jopen E et al. Biomechanical cadaver testing of a fixed-angle plate in comparison to tension wiring and screw fixation in transverse patella fractures. Injury 2012; 43: 1290-1295. doi:10.1016/j.injury.2012.04.020

[44] Wild M, Eichler C, Thelen S et al. Fixed-angle plate osteosynthesis of the patella - an alternative to tension wiring? Clin Biomech (Bristol, Avon) 2010; 25: 341-347. doi:10.1016/j. clinbiomech.2009.12.010

[45] Wild M, Thelen S, Jungbluth P et al. Fixed-angle plates in patella fractures - a pilot cadaver study. Eur J Med Res 2011; 16: 41-46

[46] Wild M, Fischer K, Hilsenbeck F et al. Treating patella fractures with a fixed-angle patella plate - A prospective observational study. Injury 2016; 47: 1737-1743. doi:10.1016/j.injury.2016. 06.018

[47] Wurm S, Augat P, Bühren V. Biomechanical assessment of locked plating for the fixation of patella fractures. J Orthop Trauma 2015; 29: e305-e308. doi:10.1097/BOT. 0000000000000309

\section{Bibliografie}

DOI https://doi.org/10.1055/a-0757-8807

Online-publiziert 01.02.2019| OP-JOURNAL 2019; 35: 156 161 @ Georg Thieme Verlag KG Stuttgart · New York ISSN 0178-1715 